\title{
Contemporary Trends of Reported Sepsis Among Maternal Decedents in Texas: A Population-Based Study
}

Lavi Oud

To view enhanced content go to www.infectiousdiseases-open.com

Received: July 23, 2015 / Published online: September 3, 2015

(C) The Author(s) 2015. This article is published with open access at Springerlink.com

\begin{abstract}
Introduction: Recent studies indicate that death certificate-based single-cause-of-death diagnoses can substantially underestimate the contribution of sepsis to mortality in the general population and among maternal decedents. There are no population-based data in the United States on the patterns of the contribution of sepsis to pregnancy-associated deaths.
\end{abstract}

Methods: We studied the Texas Inpatient Public Use Data File to identify pregnancy-associated hospitalizations with reported hospital death during 2001-2010. We then examined the annual reporting of sepsis, and that of other reported most common causes of maternal death, including hemorrhage, embolism, preeclampsia/eclampsia,

Electronic supplementary material The online version of this article (doi:10.1007/s40121-015-0086-6) contains supplementary material, which is available to authorized users.

L. Oud $(\bowtie)$

Division of Pulmonary and Critical Care Medicine, Department of Internal Medicine, Texas Tech University Health Sciences Center at the Permian Basin, Odessa, TX, USA

e-mail: lavi.oud@ttuhsc.edu cardiovascular conditions, cardiomyopathy, cerebrovascular accidents, and anesthesia complications. The annual rate of sepsis among decedents, its trend over time, and changes of its annual rank among other examined potential causes of maternal death were assessed.

Results: There were 557 pregnancy-associated hospital deaths during study period. Sepsis was reported in 131 (23.5\%) decedents. Sepsis has been increasingly reported among decedents, rising by $9.1 \% /$ year $(P=0.0025)$. The rank of sepsis, as compared to the other examined potential causes of maternal death rose from the 5 th in 2001 to 1 st since 2008 . At the end of the last decade, sepsis has been reported in $28.1 \%$ of pregnancy-associated deaths. More than one potential cause of maternal death was reported in $39 \%$ of decedents.

Conclusion: Sepsis has become the most commonly reported potential cause of death among maternal decedents in the present cohort, noted in over 1 in 4 fatal hospitalizations by the end of the last decade. Although causality cannot be inferred from administrative data, given its known contribution to maternal death, it is likely that 
sepsis plays an increasing role in fatal maternal hospital outcomes. The prevalent co-reporting of multiple potential causes of maternal death in the present cohort underscores the complexity of determining the sources of evolving rise of maternal mortality.

Keywords: Mortality; Pregnancy; Sepsis

\section{INTRODUCTION}

Sepsis has recently become the most common cause of direct maternal death in the United Kingdom (UK), as determined by the Confidential Enquiry into Maternal Deaths, involving review of decedents' medical records [1]. In the United States (US), determination of causes of maternal death is based primarily on review of death certificates, as part of the Pregnancy Mortality Surveillance System conducted by the Center of Disease Control and Preventions (CDC) [2, 3], with infections recently ranking as the third most common cause [3]. The aforementioned Surveillance System reports on causes of pregnancy-related death, that is, "a death of a woman while pregnant or within 1 year of pregnancy termination from any cause related to or aggravated by the pregnancy or its management, but not from accidental or incidental causes" [2]. This group is a subcategory of pregnancy-associated deaths, defined as the death of a woman, from any cause, while she is pregnant or within 1 year of termination of pregnancy. While forming a practical basis for national vital statistics, determination of an underlying single cause of death through death certificates is limited by well-described inaccuracies [4], which may lead to misinformed healthcare policy and research [5-7]. More specifically, determination of an association of deaths with pregnancy in the US depends on use of a specific checkbox on a death certificate to indicate that a decedent may have been pregnant up to 1 year prior to death. However, while clearly an improvement, a recent study by Horon and colleagues [8] found that only $65 \%$ of pregnancy-associated deaths were captured through use of check boxes on death certificates in Maryland.

As importantly, death certificate-based reports focus on a single cause of death due to an underlying disease which has initiated a train of events leading directly to or indirectly to death [9]. However, this reported causal information on maternal deaths lacks the broader clinical context available in medical records on conditions both in the chain events leading to death or those that may have contributed separately to maternal demise. Previous studies documented a low level of agreement between death certificate-based cause of death and the sequence of events described in medical records $[10,11]$. In addition, despite serial cause-of-death tracking, the rate of pregnancy-related mortality has been progressively rising [3], with many, if not most, events considered preventable, with related prevalent substandard care $[1,12,13]$. With the multisystem physiological changes associated with the demands of pregnancy, the risk of further deterioration may be increased in obstetric patients with decompensated new or chronic illness, even when not directly related to or aggravated by pregnancy. Thus, broader characterization of the scope of co-existing or evolving conditions among maternal decedents may better inform health care policy and clinical practice to identify areas for intervention needed to effectively disrupt the chain of events leading to death.

Because infections can often complicate more chronic conditions, the former are more likely to be underreported in death certificates. 
This was recently demonstrated in a study of the general US population by Govindan and colleagues [14], where the top three death certificate-based causes of death matched, as expected, national reports (heart disease, malignancy, and cerebrovascular disease). However, hospital data showed that infections were the most common diagnoses of same terminal hospitalizations [14].

Whether an infection contributes to or causes patient's death, it is the dysregulated systemic response to infection, often with resultant organ failure, namely sepsis, that is the key driver of patients' morbidity and mortality $[15,16]$. Indeed, a recent report by Liu and colleagues [17] demonstrated that sepsis may have contributed to one in every two to three hospital deaths in the general US population. However, the mortality from sepsis associated with pregnancy may be underreported in clinical studies, partly because in many studies sepsis was neither defined, nor classified as an infectious cause of death [18]. Nevertheless, when causes of maternal death were directly examined, it was sepsis, rather than infection that was considered causal [1]. Similarly, sepsis, rather than infection, has been examined among causes of severe maternal morbidity [19]. On the other hand, although reported causes of maternal deaths by the Pregnancy Mortality Surveillance System include an infection category, under which there are subcategories of specific sites of infection, sepsis appears as another infectious subcategory. Thus, in a recent report by Creanga et al. [3] sepsis constituted only $32 \%$ of all infectious causes of death, although sepsis is not a site-specific condition and it would be implausible that sepsis was not present in the remainder maternal decedents judged to die due to an infectious cause. The occurrence of sepsis at a population-level among hospitalized maternal decedents in the US has not been reported, to our knowledge.

Our aim was to examine the contemporary patterns of the burden of sepsis in a population-level cohort of maternal decedents in Texas during terminal pregnancy-associated hospitalizations and its relative rank compared to the most common reported causes of maternal death.

\section{METHODS}

\section{Setting and Data Sources}

We performed a retrospective, population-based cohort study of pregnancy-associated mortality during 2001-2010, using the Texas Inpatient Public Use Data File (TIPUDF), a longitudinal data set maintained by the Texas Department of State Health Services [20]. The data set includes detailed de-identified inpatient discharge data from all state-licensed hospitals, with the exception of those exempt by state statute from reporting to the Texas Health Care Information Collection. Exempt hospitals include (a) those that do not seek insurance payment or government reimbursement and (b) selected rural providers, based on bed number and local county population. The facilities included in the mandated report account for $93 \%$ to $97 \%$ of all hospital discharges. The TIPUDF data set includes demographic, clinical, resource utilization, and outcome information. The data set includes up to 25 discharge diagnoses, and up to 25 procedures, coded using the International Classification of Diseases, Ninth Revision, Clinical Modification (ICD-9-CM). Because we used a publicly available, de-identified data set, this study was determined to be exempt from formal review by the Texas Tech Health Sciences Center Institutional Review Board. 
This article does not involve any new studies with human or animal subjects performed by any of the authors.

\section{Study Population}

We used ICD-9-CM codes (please see Table S1 in the supplementary material for details) to identify Texas residents with pregnancy-associated hospitalizations between 2001 and 2010. Pregnancy-associated hospitalizations with reported hospital death were analyzed.

\section{Data Collection}

We collected data on patients' age, race (categorized as non-Hispanic black [black], non-Hispanic white [white], Hispanic, and other), health insurance (categorized as private, Medicaid, uninsured, and other), and type of pregnancy-associated hospitalization. We categorized the type of pregnancy-associated hospitalizations into the following mutually exclusive, hierarchical groups, using pregnancy-associated ICD-9-CM codes: (a) miscarriage (pregnancies with abortive outcome, excluding induced abortion), (b) induced abortion, (c) delivery (based on the approach described by Kuklina et al. [21]), (d) post-partum (hospitalizations with an ICD-9-CM code for puerperal complications, without pregnancy-related diagnosis codes of groups a-c), and (e) antepartum (hospitalization with pregnancy-related diagnosis, but without those pregnancy-related diagnosis codes included in groups a-d). We then examined the cohort of maternal decedents for reporting of sepsis and other specific systemic conditions, diseases or complications of therapy, modeled on the reported categories by the Pregnancy Mortality
Surveillance System as causes of maternal death [3]. These conditions included primary or secondary diagnoses of: hemorrhage, embolism, preeclampsia/eclampsia, cardiovascular conditions, cardiomyopathy, cerebrovascular accident, and anesthesia complications (please see Table S2 in the supplementary material for details). The case definition of sepsis was modeled on the coding system reported by Lagu et al. [22]. Specifically, sepsis was defined as primary or secondary diagnosis codes of either an infectious process (please see Table S3 in the supplementary material for details) or a specific ICD-9-CM code 995.91 (systemic inflammatory response syndrome due to an infectious process without organ failure). A subcategory of severe sepsis was defined as: (a) the presence of any of the infectious process under the definition of sepsis (excluding ICD-9-CM code 995.91), combined with ICD-9-CM codes of at least one organ failure (please see Table S4 in the supplementary material for details), or (b) an ICD-9-CM code for either septic shock (785.52) or systemic inflammatory response syndrome due to an infectious process with organ failure (995.92).

\section{Outcomes}

The primary outcome was the annual rate of reported sepsis among maternal decedents. The secondary outcome was the annual rank of sepsis, as compared to the remainder examined potential causes of maternal death.

\section{Data Analysis}

We report on pregnancy-associated deaths, that is, focusing on decedents of pregnancy-associated hospitalizations, without determination whether death has been due to any cause related to or aggravated by the 
pregnancy or its management [2]. Because administrative data sets preclude assessment of causality of reported clinical conditions or procedures, we examined reporting of sepsis and other potential causes of maternal death as contributing conditions (termed as contributor(s) in the remainder of the manuscript) among maternal decedents. However, while the presence of sepsis or other examined conditions is unlikely to be inconsequential, the magnitude of contribution of each could not be inferred. Rather, we describe pregnancy-associated deaths with sepsis or any of the other examined contributors.

Eleven hospitalizations associated with miscarriage/induced abortion could not be adequately classified to only one group (that is, either miscarriage or induced abortion), because their only pregnancy-associated ICD-9-CM code was 639.XX (complications following abortion and ectopic and molar pregnancies). We, thus, combined miscarriage and induced abortion into a single group.

Group data are reported as numbers (percentages) for categorical variables. Linear regression of log-transformed annual rate of sepsis among maternal decedents was used to examine trends over study years.

All statistical analyses were performed using MedCalc version 15.6 (MedCalc Software, Ostend, Belgium) and SAS version 9.3 (SAS Institute, Cary, NC, USA). A 2-sided $P$ value $<0.05$ was considered significant.

\section{RESULTS}

There were 4,060,659 pregnancy-associated hospitalizations with 557 deaths during the 2001-2010 period. The demographic characteristics, health insurance, and the category of pregnancy-associated hospitalization of maternal decedents are detailed in Table 1. Women aged 35 years or older comprised $22.3 \%$ of maternal decedents. Most maternal deaths involved Hispanic women (40.8\%), with Medicaid being the most common type of health insurance (48.1\%). Maternal death occurred predominantly during delivery hospitalizations (57.6\%), with

Table 1 The demographic characteristics, health insurance and categories of pregnancy-associated hospitalizations among maternal decedents

\begin{tabular}{|c|c|}
\hline Group & $\begin{array}{l}\text { Maternal decedents } \\
(n=557)\end{array}$ \\
\hline \multicolumn{2}{|l|}{ Age (years), $n[\%]$} \\
\hline$<18$ & $22(3.9)$ \\
\hline $18-34$ & $411(73.8)$ \\
\hline$\geq 35$ & $124(22.3)$ \\
\hline \multicolumn{2}{|l|}{ Race/ethnicity, $n$ (\%) } \\
\hline Hispanic & $227(40.8)$ \\
\hline Black & $127(22.8)$ \\
\hline White & $156(28.0)$ \\
\hline Other & $47(8.4)$ \\
\hline \multicolumn{2}{|l|}{ Health insurance, $n(\%)$} \\
\hline Private & $169(30.3)$ \\
\hline Medicaid & $268(48.1)$ \\
\hline Medicare & $16(2.9)$ \\
\hline Uninsured & $88(15.8)$ \\
\hline Other $^{\mathrm{a}}$ & $16(2.9)$ \\
\hline \multicolumn{2}{|c|}{ Category of hospitalization, $n(\%)$} \\
\hline Miscarriage/abortion & $61(11.0)$ \\
\hline Delivery & $321(57.6)$ \\
\hline Postpartum & $100(18.0)$ \\
\hline Antepartum & $75(13.5)$ \\
\hline
\end{tabular}


postpartum hospitalizations (18.0\%) being the second most common category.

The annual occurrence of sepsis and the other examined contributors to pregnancy-associated death are detailed in Table 2. Sepsis was increasingly reported among maternal decedents, with its rate rising by $9.1 \% /$ year $\quad(P=0.0025)$. Severe sepsis accounted for $84.7 \%$ of all sepsis events. When compared with other examined contributors, sepsis ranked 5th in 2001, rising progressively and becoming since 2008 the most common condition among all examined categories. More than contributor to pregnancy-associated mortality was reported in 217 (39\%) decedents.

\section{DISCUSSION}

We found that sepsis has been increasingly reported among pregnancy-associated deaths in the present cohort. In addition, by the end of the last decade sepsis has contributed to over one in four pregnancy-associated deaths, becoming the most common contributor among the examined conditions.

The present population-based cohort focusing specifically on maternal decedents is, to our knowledge, the largest reported to date. Although numerous studies of obstetric patients included data on maternal decedents, both through direct review of medical records [12, $23,24]$ and using administrative data [25-27], the investigators focused on single causes of death of decedents [12, 23] or reported conditions associated with combined maternal morbidity and mortality [24-27] without systematic examination of overall occurrence of common potential causes of maternal death. Because we studied the contribution of the most commonly reported causes of maternal death among all pregnancy-associated deaths during women's terminal hospitalization, our findings are not directly comparable to prior reports on maternal decedents. Of note, the population-based study by Mhyre et al. [27] cited earlier described, using administrative data, various conditions reported among maternal hospitalizations with in-hospital cardiac arrest as "proximate potential etiology" of maternal cardiac arrest, including sepsis and many of the most commonly reported causes of pregnancy-related death. However, the investigators reported these conditions only for all those sustaining cardiac arrest, of whom $59 \%$ survived hospitalization.

Women aged 18-34 years formed the most common group of maternal decedents, similar to the general obstetric population. However, those aged 35 years or older were overrepresented, accounting for more than 1 in 5 of pregnancy-associated deaths, in line with comparable findings in national reports on pregnancy-related deaths [3]. Hispanic women were the most common ethnic group in the present cohort, in line with state's obstetric population [28]. However, both Hispanic and white decedents were underrepresented, while black women were represented twofold higher their share among live births the state [28], the latter extending data on high pregnancy-related mortality in the black population in the US [3].

The health insurance of maternal decedents in the US has not been previously reported at a population level, to our knowledge. Medicaid and private insurance, the most common categories in the present study, represent the first (51.4\%) and second (33.3\%) most common payers for pregnancy-associated hospitalizations in the state [29]. However, both were slightly underrepresented among pregnancy-associated deaths. It may be hypothesized that the observed differences may have been related to better and consistent access to healthcare and specifically prenatal 


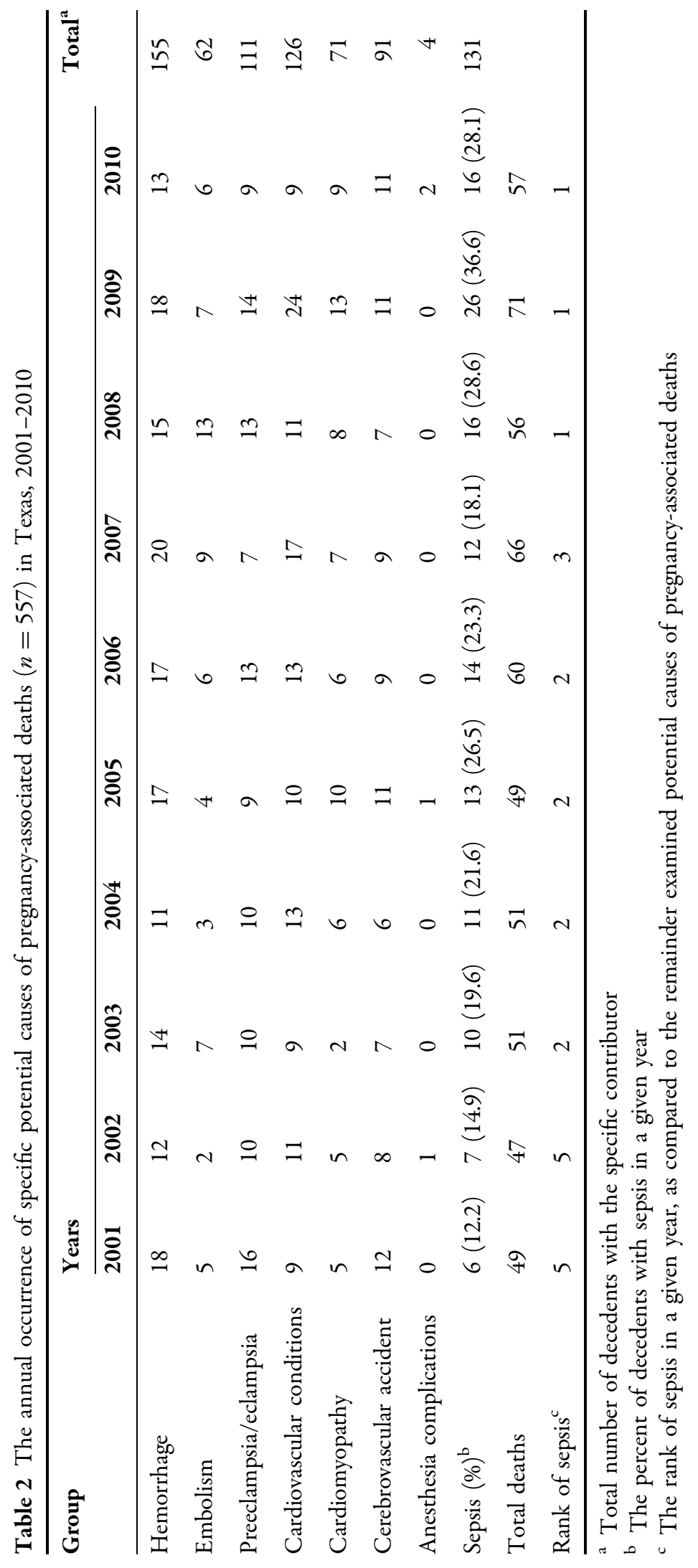


care among the insured. On the other hand Medicare insurance was used sevenfold more often by maternal decedents versus its use during all pregnancy-associated hospitalizations in the state (0.4\%) [29], likely reflecting a subgroup with substantial disability due to chronic medical problems, which would be expected to increase the risk of adverse pregnancy-associated outcomes. Finally, lack of health insurance occurred nearly 2.5 -fold higher among maternal decedents than among all pregnancy-associated hospitalizations in the state (6.7\%) [29], underscoring the adverse impact of likely lack of adequate and timely obstetric and general medical care, especially in vulnerable populations. While lack of health insurance is well known to have adverse health effects [30, 31], broader characterization of insurance state of maternal decedents can further inform future healthcare policy.

Our findings on the categories of pregnancy-associated hospitalizations are not directly comparable to the national reports of pregnancy-related deaths. However, our findings that the majority of pregnancy-associated deaths involved delivery hospitalizations are comparable to national reports where live births (64.5\%) were the most common category [3]. On the other hand, hospitalizations associated with miscarriage and induced abortion were nearly twice as common in our cohort, as compared to national reports on pregnancy-related mortality [3]. The source of the difference is unclear. However, the outcome of pregnancy could not be determined in about $10 \%$ of pregnancy-related deaths in a recent report [3], and may have contributed to the observed difference. Of note, nearly one in five of pregnancy-associated deaths occurred during separate postpartum hospitalizations. Recent data indicate that postpartum hospitalizations are associated with much higher risk of severe maternal morbidity than delivery hospitalizations. In a study by Callaghan and colleagues [32], the investigators found in a national US sample that severe maternal morbidity occurred at a 14-fold higher rate among postpartum hospitalizations than among delivery hospitalizations.

Sepsis has contributed to up to more than one in three pregnancy-associated deaths and over one in four at the end of last decade. Although sepsis has been examined in studies of maternal death $[25,27]$, there were no reports on its presence among decedents judged to die due to other causes. However, while determination of a single cause of death is essential for epidemiological studies of populations, the primary initiating causes of death are often complicated subsequently by various factors, whose effective control may alter otherwise fatal outcomes. The care of septic patients is often inadequate in the general population [33] and was reported to be substandard in the majority of maternal decedents [1, 34], mostly due to inadequate early recognition and lack of timely effective care. In addition, the majority of maternal deaths are considered preventable $[1,12,13]$. Thus, moving beyond a single-cause-of-death approach, studies on the broad scope of potential contributors to maternal mortality may inform the formulation of better targeted preventive and intervention measures that may reduce pregnancy-associated mortality.

When studied, in the general population sepsis was found to contribute to between one in two to one in three hospital deaths [17]. There are several possible sources for the difference in the extent of contribution of sepsis to patient death between maternal decedents and the general population, as described by Liu et al. [17]. First, the majority 
of septic patients in the general population are elderly [22], with high burden of chronic illness and immunosuppressive states, while obstetric patients are generally healthy. In addition, we have used a conservative approach to identify sepsis, based mostly on the diagnoses of septicemia with or without organ failure [22, 35]. On the other hand, Liu and colleagues employed a broad case definition of sepsis, including numerous sites of infection [36]. However, while the latter approach was originally validated when coupled with organ failure to identify severe sepsis, its role remains uncertain when substituting a diagnosis of infection for the presence of sepsis. Thus, sepsis has contributed to a substantial proportion of pregnancy-associated deaths in the present cohort even with use of a conservative case definition. The noted case definition of sepsis versus severe sepsis used by Liu et al. [17] also likely explains their findings that the majority of deaths with sepsis occurred among patients without severe sepsis, in contrast to the opposite in our cohort. However, it would be expected that organ failure will commonly develop when sepsis contributes to death.

Sepsis was increasingly reported among maternal decedents during the last decade, reflecting rising incidence of sepsis in the obstetric population in the state [37] and nationally [38]. Oud et al. [37] have recently reported that the incidence of pregnancy-associated severe sepsis in Texas rose by $9.7 \%$ /year between 2001 and 2010 with corresponding increased sepsis-associated mortality rate from 1.1 to 3.3 deaths per 100,000 Total Estimated Pregnancies [37]. Although the rise of reported events of pregnancy-associated severe sepsis may be considered to reflect over-coding and inclusion of less severe sepsis events, this hypothesis was not supported by the simultaneous rise in the rate of admission to intensive care unit, resource utilization, and unchanged case fatality [37]. Alternatively, the observed changes in reported events of severe sepsis the obstetric population could have resulted from higher awareness by clinicians, especially with a global initiative such as the Surviving Sepsis Campaign [33], rather than actual increase in incidence. However, sepsis among pregnant patients has also been rising in the preceding decades [35]. Moreover, a suggestion of increase clinicians' awareness of maternal sepsis is not supported by the lack of decrease in case fatality over time among hospitalizations with pregnancy-associated severe sepsis [37], in contrast with the consistently documented decrease in case fatality of severe sepsis in the general population [39]. In addition, sepsis has recently become the most common cause of direct maternal death in the UK [1], based on the direct examination of clinical records, which further showed prevalent lack timely recognition and intervention in maternal decedents with sepsis. Although the incidence of pregnancy-associated sepsis and sepsis-associated mortality were examined in other reports, only few non-US studies evaluated longitudinal population-level trends of sepsis associated death in the obstetric population. Recent reports from the Netherlands [40] and France [41] noted no significant change in maternal mortality rate from infections or sepsis. However, both studies included very small number of decedents and the French study examined only infections as direct cause of death [41]. No non-US data were reported, to our knowledge, on longitudinal, population-based trends of the incidence of pregnancy-associated sepsis. Of note, no longitudinal population-based studies of sepsis-associated deaths in the obstetric 
population were reported from developing countries. Indeed, investigators have commented on the overall underestimation of maternal death in areas where the burden of sepsis is greatest [42]. It has been suggested that the increasing maternal age, obesity, chronic illness, use of cesarean section, and other invasive procedures in the obstetric population has contributed to the observed rise of pregnancy-associated sepsis [43]. In addition, increasing virulence of infecting microorganisms and increasing resistance to antimicrobials may have contributed to the observed incidence changes. Assuming actual rise in the incidence of maternal sepsis, addressing the underlying causes may help to reduce fatal maternal outcomes.

We chose to examine reporting of other categories of specific system, disease, or intervention-related causes of maternal death reported in the US [3] to better characterize the relative contribution of sepsis to pregnancy-associated mortality. The rank of sepsis as contributor to pregnancy-associated deaths among the other examined contributors rose progressively during study period, becoming the most common contributor over the last 3 years of the past decade. However, although the rank of sepsis increased consistently over time, the small number of decedents under each category of potential causes of maternal death in a given year limits the precision of our rank examination. Thus, our findings require further corroboration in other populations.

The contribution of sepsis to patients' death may have been underestimated in both the general population and among maternal decedents. Severe sepsis in the general population has been recently reported to be associated with up to nearly 370,000 annual deaths in the US [44], making it, if causative, among the most common causes of death in the country. However, neither sepsis nor severe sepsis is included as the tracked cause of death in national statistics. Even when considered causative by clinicians, entering sepsis as cause of death was proven challenging in some locations [45]. The scope of underestimation of sepsis as cause of death has been recently demonstrated in a study by Ong and colleagues [46], noting that reporting of sepsis as cause of death has increased from $3.7 \%$ to $20 \%$ with clinician training, while a decline was noted when no training was provided.

In the obstetric population, imprecise terminology in clinical practice and research reports may have contributed to underestimation of the role of sepsis among maternal decedents [18]. In a recent study by Horon and colleagues [8], comparing death certificate-based causes

of pregnancy-associated death to a broader source of clinical data, infectious causes of death were the most common underreported medical condition, included in only $50 \%$ of corresponding death certificates. However, as noted earlier, when maternal deaths were systematically reviewed by the Confidential Enquiry into Maternal Deaths, sepsis was increasingly common, becoming the most common direct cause of maternal death in the UK [1]. Thus, even when reported, sepsis is likely underestimated among infectious causes of death [3]. A study of administrative data cannot quantify the degree to which sepsis has contributed to pregnancy-associated death among affected women. However, pregnancy-associated sepsis can be rapidly lethal [1, 34]. Given the prevalent substandard care of maternal sepsis [1, 34], the increasing reporting of sepsis among decedents in our cohort underscores the need to improve clinicians' and healthcare systems' 
performance to assure timely recognition and care in order to disrupt the sequence of lethal events, regardless of whether sepsis is the primary cause or a separate contributor. Our not unexpected finding that more than one of the examined contributors to maternal death was present in 39\% of decedents underscores the challenges and limitations of the current single cause of death reporting when striving to apply findings to bedside clinical practice.

Our findings should be considered in the context of several limitations. First, the retrospective design of the present study and use of an administrative data set with their attendant limitations affects the interpretation of our results. However, the rarity of pregnancy-associated death in the US can be a challenge for alternative population-based approaches.

In addition, our cohort was based on identified pregnancy-associated hospitalizations. Thus, we may have omitted maternal deaths among hospitalizations not coded as pregnancy-associated. However, pregnancy-associated hospitalizations were the most common category of hospitalization [29] in Texas, with expected high clinician and coder familiarity. In addition, we have likely omitted hospitalizations beyond the postpartum period, but less than 1 year following end of hospitalization, and could not examine pregnancy-associated deaths occurring outside the hospital. Nevertheless, similar methodological limitations have affected other population-based studies of maternal death based on administrative data [23, 25-27], as well as studies of severe maternal morbidity [19]. Finally, the proportions of the various categories of pregnancy-associated hospitalizations in the present study were comparable to those reported by other investigators [47].
The optimal ICD code-based approach to identify patients with sepsis and specifically severe sepsis in administrative data sets remains unsettled. A recent study by Gaieski and colleagues [44], using a national data set, demonstrated nearly 3.5 -fold difference in the number of identified severe sepsis hospitalizations, and marked variability in the number of failing organs and case fatality between 4 different code-based methods. We chose a conservative approach to identify sepsis hospitalizations, in part because it produces for the severe sepsis subset a distribution of the number of failing organs comparable to epidemiological chart-based studies of severe sepsis in the general population [48, 49], and we observed comparable findings in a prior study [37], being in line with distribution of the number of failing organs in a chart-based study of pregnancy-associated severe sepsis [50], supporting our case identification approach. Nevertheless, we cannot exclude a possibility of underestimating occurrence of sepsis hospitalizations. As noted by Gaieski et al. [44], the various approaches to identify severe sepsis in the general population demonstrated similar upward trends over time. Thus, it is unlikely that the observed increased occurrence of sepsis in the present cohort is due our case identification approach.

We have identified the other examined potential causes of maternal death based on specific ICD-9-CM codes. Thus, we cannot exclude possible misclassification. However, our approach for identification of each of the examined conditions was based on very broad code array and is similar to that reported by other investigators $[20,25,27]$. Thus, a systematic bias in the annual reporting of the examined conditions or general underestimation, as compared to our case definition of sepsis, is unlikely. 
Finally, we examined pregnancy-associated deaths in Texas due to its large and diverse population, with a high-quality, longitudinal hospital discharge data set. However, population characteristics, clinical practice patterns, and the resultant contribution of sepsis to maternal death and relative to the other examined potential causes of maternal death may vary across states and nationally. Further studies on the role of sepsis in pregnancy-associated death are warranted in other healthcare environments.

\section{CONCLUSIONS}

The present study is, to our knowledge, the first population-based examination to date of the contemporary patterns of reporting of sepsis in pregnancy-associated hospital deaths. The rate of reported sepsis among maternal decedents rose $130 \%$ during study period. Sepsis has become the most commonly reported condition among the examined potential causes of maternal mortality, contributing to over one in four pregnancy-associated deaths in the present cohort by the end of the last decade. The causal role and the degree of contribution of sepsis and the other examined contributors to maternal death in individual women cannot be inferred from administrative data. However, given its well-known contribution to maternal mortality [1] and morbidity [19], it is likely that sepsis plays an increasing role in fatal maternal hospital outcomes. The prevalent co-reporting of multiple potential known causes of maternal death in the present cohort underscores the complexity of determining the factors underlying the evolving trends of increasing maternal mortality. Further studies are warranted in other populations to better characterize the role of sepsis in pregnancy-associated mortality in order to limit its adverse impact in the obstetric population. Finally, future studies involving direct examination of the clinical data of all maternal decedents, following the lead in the UK [1], are key to better understand the relative role of predisposing conditions-, pathogen-, clinician-, health care systems-, and patient-related factors driving the observed trends.

\section{ACKNOWLEDGMENTS}

No funding or sponsorship was received for this study or publication of this article. The author meets the International Committee of Medical Journal Editors (ICMJE) criteria for authorship for this manuscript, takes responsibility for the integrity of the work as whole, and has given final approval for the version published. The author would like to thank Phillip Watkins, MS, for his help with parts of data acquisition and management.

Conflict of interest. L. Oud declares no conflict of interest.

Compliance with ethics guidelines. Because we studied publicly available, de-identified data set, this study was determined to be exempt from formal review by the Texas Tech Health Sciences Center Institutional Review Board. This article does not contain any new studies with human or animal subjects performed by the author.

Open Access. This article is distributed under the terms of the Creative Commons Attribution-NonCommercial 4.0 International License (http://creativecommons.org/licenses/ by-nc/4.0/), which permits any noncommercial use, distribution, and reproduction in any medium, provided you give appropriate credit 
to the original author(s) and the source, provide a link to the Creative Commons license, and indicate if changes were made.

\section{REFERENCES}

1. Cantwell R, Clutton-Brock T, Cooper G, et al. Saving mothers' lives: reviewing maternal deaths to make motherhood safer: 2006-2008. The eighth report of the confidential enquiries into maternal deaths in the United Kingdom. BJOG. 2011;118(suppl 1):1-203.

2. Berg C, Danel I, Atrash H, Zane S, Bartlett L, editors. Strategies to reduce pregnancy-related deaths: from identification and review to action. Atlanta: Centers for Disease Control and Prevention; 2001. http://www.cdc.gov/reproductivehealth/Products Pubs/PDFs/Strategies_taged.pdf. Accessed 12 May 2015.

3. Creanga AA, Berg CJ, Syverson C, Seed K, Bruce FC, Callaghan WM. Pregnancy-related mortality in the United States, 2006-2010. Obstet Gynecol. 2015;125:5-12.

4. Messite J, Stellman SD. Accuracy of death certificate completion: the need for formalized physician training. JAMA. 1996;275:794-6.

5. Johansson LA, Westerling R. Comparing Swedish hospital discharge records with death certificates: implications for mortality statistics. Int J Epidemiol. 2000;29:495-502.

6. Hinchliffe SR, Abrams KR, Lambert PC. The impact of under and over-recording of cancer on death certificates in competing risks analysis: a simulation study. Cancer Epidemiol. 2013;37:11-9.

7. Johns LE, Madsen AM, Maduro G, Zimmerman R, Konty K, Begier E. A case study of the impact of inaccurate cause-of-death reporting on health disparity tracking: New York City premature cardiovascular mortality. Am J Public Health. 2013;103:733-9.

8. Horon IL, Cheng D. Effectiveness of pregnancy check boxes on death certificates in identifying pregnancy-associated mortality. Public Health Rep. 2011;126:195-200.

9. Instruction for clarifying the underlying cause of death. National Vital Statistics System. Centers for Disease Control and Prevention; 2015. http://www. cdc.gov/nchs/data/dvs/2a_2015.pdf. Accessed 3 Feb 2015.
10. Lloyd-Jones DM, Martin DO, Larson MG, Levy D. Accuracy of death certificates for coding coronary artery disease as cause of death. Ann Intern Med. 1998;129:1020-6.

11. Hoff CJ, Ratard R. Louisiana death certificate accuracy: a concern for the public health. J La State Med Soc. 2010;162:352-3.

12. Main EK, Mccain CL, Morton CH, Holtby S, Lawton ES. Pregnancy-related mortality in California: causes, characteristics, and improvement opportunities. Obstet Gynecol. 2015;125:938-47.

13. Gaskin IM. Maternal death in the United States: a problem solved or a problem ignored. J Perinat Educ. 2008;17:9-13.

14. Govindan S, Shapiro L, Langa KM, Iwashyna TJ. Death certificates underestimate infections as proximal causes of death in the US. PLoS One. 2014;9:e97714.

15. Bone RC, Balk RA, Cerra FB, et al. Definitions of sepsis and organ failure and guidelines for the use of innovative therapies in sepsis. The ACCP/SCCM Consensus Conference Committee. American College of Chest Physicians/Society of Critical Care Medicine. Chest. 1992;101:1644-55.

16. Levy MM, Fink MP, Marshall JC, et al; SCCM/ ESICM/ACCP/ATS/SIS. 2001 SCCM/ESICM/ACCP/ ATS/SIS International Sepsis Definitions Conference. Crit Care Med. 2003;31:1250-6.

17. Liu V, Escobar GJ, Greene JD, et al. Hospital deaths in patients with sepsis from 2 independent cohorts. JAMA. 2014;312:90-2.

18. Guinn DA, Abel DE, Tomlinson MW. Early goal directed therapy for sepsis during pregnancy. Obstet Gynecol Clin N Am. 2007;34:459-79.

19. Callaghan WM, Mackay AP, Berg CJ. Identification of severe maternal morbidity during delivery hospitalizations, United States, 1991-2003. Am J Obstet Gynecol. 2008;199:113.e1-133.e8.

20. Texas inpatient public use data file. Texas Health Care Information Center for Health Statistics. Texas department of state health services. http://www. dshs.state.tx.us/thcic/hospitals/Inpatientpudf.shtm. Accessed 10 Feb 2015.

21. Kuklina EV, Whiteman MK, Hillis SD, et al. An enhanced method for identifying obstetric deliveries: implications for estimating maternal morbidity. Matern Child Health J. 2008;12:469-77.

22. Lagu T, Rothberg MB, Shieh M, Pekow PS, Steingrub JS, Lindenauer PK. Hospitalizations, costs and 
outcomes of severe sepsis in the United States 2003 to 2007. Crit Care Med. 2012;40:754-61.

23. Clark SL, Belfort MA, Dildy GA, Herbst MA, Meyers JA, Hankins GD. Maternal death in the 21st century: causes, prevention, and relationship to cesarean delivery. Am J Obstet Gynecol. 2008;199:36.1e-36.e5.

24. Goffman D, Madden RC, Harrison EA, Merkatz IR, Chazotte CC. Predictors of maternal mortality and near-miss maternal morbidity. J Perinatol. 2007;27:597-601.

25. Bateman BT, Mhyre JM, Hernandez-Diaz S, et al. Development of comorbidity index for use in obstetric patients. Obstet Gynecol. 2013;122:957-65.

26. Mhyre JM, Bateman BT, Leffert LR. Influence of patient comorbidities on the risk of near-miss maternal morbidity or mortality. Anesthesiology. 2011;115:963-72.

27. Mhyre JM, Tsen LC, Einav S, Kuklina EV, Leffert LR, Bateman BT. Cardiac arrest during hospitalization for delivery in the United States, 1998-2011. Anesthesiology. 2014;120:810-8.

28. Vital statistics annual reports. Texas department of state health services. https://www.dshs.state.tx.us/ chs/vstat/annrpts.shtm. Accessed 5 Mar 2015.

29. Facts and figures: statistics on hospital-based care in Texas. Texas Health Care Information Collection. DSHS Publication \# E87-11648; 2009. http://www. dshs.state.tx.us/thcic/publications/hospitals/statisti calreports.shtm. Accessed 17 Feb 2015.

30. Shen JJ, Wei H. Adverse maternal outcomes for women with different health insurance statuses in Nevada. Nev J Public Health. 2008;5(1):Article 5. http://digitalscholarship.unlv.edu/njph/vol7/iss1/4.

31. Curtin SC, Osterman MJ, Uddin SF, Sutton SR, Reed PR. Source of payment for delivery: births in a 33-state and district of Columbia reporting area, 2010. Nat Vital Stat Rep. 2013;62:1-20.

32. Callaghan WM, Creanga AA, Kuklina EV. Severe maternal morbidity among delivery and postpartum hospitalizations in the United States. Obstet Gynecol. 2012;120:1029-36.

33. Levy MM, Rhodes A, Phillips GS, et al. Surviving Sepsis Campaign: association between performance metrics and outcomes in a 7.5-year study. Crit Care Med. 2015;43:3-12.

34. Kramer HM, Schuttle JM, Zwart JJ, Schuitemaker NW, Steegers EA, van Roosmalen J. Maternal mortality and severe morbidity from sepsis in the
Netherlands. Acta Obstet Gynecol Scand. 2009;88:647-53.

35. Martin GS, Mannino DM, Eaton S, Moss M. The epidemiology of sepsis in the United States from 1979 through 2000. N Engl J Med. 2003;348:1546-54

36. Angus DC, Linde-Zwirble WT, Lidicker J, Clermont G, Carcillo J, Pinsky MR. Epidemiology of severe sepsis in the United States: analysis of incidence, outcomes, and associated costs of care. Crit Care Med. 2001;29:1303-10.

37. Oud L, Watkins P. Evolving trends in the epidemiology, resource utilization, and outcomes of pregnancy-associated severe sepsis: a population-based cohort study. J Clin Med Res. 2015;7:400-16.

38. Bauer ME, Bateman BT, Bauer ST, Shanks AM, Mhyre JM. Maternal sepsis mortality and morbidity during hospitalization for delivery: temporal trends and independent predictors for severe sepsis. Anesth Analg. 2013;117:944-50.

39. Stevenson EK, Rubenstein AR, Radin GT, Wiener RS, Walkey AJ. Two decades of mortality trends among patients with severe sepsis: a comparative meta-analysis. Crit Care Med. 2014;42:625-31.

40. Schutte JM, Steegers EA, Schuitemaker NW, Santema JG, de Boer K, Pel M, Vermeulen G, Visser W, van Roosmalen J, Netherlands Maternal Mortality Committee. Rise in maternal mortality in the Netherlands. BJOG. 2010;117:399-406.

41. Benhamou D, Chassard D, Mercier FJ, Bouvier-Colle MH. The seventh report of the confidential enquiries into maternal deaths in the United Kingdom: comparison with French data. Ann Fr Anesth Reanim. 2009;28:38-43.

42. What you count is what you target: the implication of maternal death classification for tracking progress towards reducing maternal mortality in developing countries. Bull World Health Organ 2010;88:147-53.

43. Barton JR, Sibai BM. Severe sepsis and septic shock in pregnancy. Obstet Gynecol. 2012;120:689-706.

44. Gaieski DF, Edwards JM, Kallan MJ, Carr BG. Benchmarking the incidence and mortality of severe sepsis in the United States. Crit Care Med. 2013;41:1167-74.

45. Wexelman BA, Eden E, Rose KM. Survey of New York City resident physicians on cause of death reporting, 2010. Prev Chronic Dis. 2013;10:E76. 
46. Ong P, Gambatese M, Begier E, Zimmerman R, Soto A, Madsen A. Effect of cause-of-death training on agreement between hospital discharge diagnoses and cause of death reported, inpatient hospital deaths, New York City, 2008-2010. Prev Chronic Dis. 2015;12:E04.

47. Waters TP, Bailit JL. Obstetric and non-obstetric indications for admission in the antepartum and postpartum periods in pregnancy. J Women's Health Care. 2012;1:104.

48. Ferrer R, Martin-Loeches I, Phillips G, et al. Empiric antibiotic treatment reduces mortality in severe sepsis and septic shock from the first hour: results from a guideline-based performance improvement program. Crit Care Med. 2014;42:1749-55.

49. Lagu T, Rothberg MB, Shieh MS, Pekow PS, Steingrub JS, Lindenauer PK. What is the best method for estimating the burden of severe sepsis in the United States? J Crit Care. 2012;27:e1-9.

50. Snyder CC, Barton JR, Habli M, Sibai BM. Severe sepsis and septic shock in pregnancy: indications for delivery and maternal and perinatal outcomes. J Matern Fetal Neonatal Med. 2013;26:503-6. 